\title{
Probing student reasoning in relating relative phase and quantum phenomena
}

\author{
Tong Wan $\odot,{ }^{1, *}$ Paul J. Emigh, ${ }^{2}$ and Peter S. Shaffer ${ }^{3}$ \\ ${ }^{1}$ Department of Physics, University of Central Florida, Orlando, Florida 32816, USA \\ ${ }^{2}$ Department of Physics, Oregon State University, Corvallis, Oregon 97330, USA \\ ${ }^{3}$ Department of Physics, University of Washington, Seattle, Washington 98195, USA
}

(Received 17 May 2019; published 31 October 2019; corrected 24 February 2021)

\begin{abstract}
In quantum mechanics, probability amplitudes are complex numbers and the relative phases between the terms in superposition states have measurable effects. This article describes an investigation into sophomore- and junior-level students' reasoning patterns in relating relative phases and real-world quantum phenomena. The investigation involved one observational experiment and three testing experiments, during which we formulated and tested three hypotheses that allow us to gain insights into why students have difficulty recognizing the measurable effects of relative phases. We found that, in both spin-1/2 and infinite square well contexts, many students do not recognize that quantum states differing only by a relative phase are experimentally distinguishable. Moreover, student ability to recognize the measurable effects of relative phase does not improve when given (i) a task that specifically prompts students to compare the probabilities for a particular observable and (ii) a task that does not require taking inner products or changing basis. We also examined the extent to which lacking proficiency with complex numbers may have hindered student understanding of relative phase. The data indicate that most students are proficient with complex numbers. These findings suggest that many students do not, in fact, recognize the purpose of using complex numbers in superposition states. We discuss possible explanations for why students do not seem to recognize this purpose, and we also provide suggestions for future avenues of research.
\end{abstract}

DOI: 10.1103/PhysRevPhysEducRes.15.020139

\section{INTRODUCTION}

Relative phase is one of the fundamental concepts in quantum mechanics. Probability amplitudes are complex numbers that are often presented as the product of a real magnitude and a complex exponential. Different relative phases between the different probability amplitudes in a superposition state then give rise to (measurable) differences in some probabilities. One canonical example is the SternGerlach experiment. The relative phase between the various spin- $1 / 2$ basis states can be determined by measuring various components of the spin. Furthermore, relative phase underlies the measurable impacts of quantum time dependence, which research has found is difficult for many students [1-4]. When a superposition state evolves in time, the relative phase also changes in time, resulting in timedependent probabilities. Therefore, a solid understanding of the measurable effects of relative phases is a key step to grasping the fundamentals in quantum mechanics.

\footnotetext{
*tongwan531@gmail.com
}

Published by the American Physical Society under the terms of the Creative Commons Attribution 4.0 International license. Further distribution of this work must maintain attribution to the author(s) and the published article's title, journal citation, and DOI.
Making a connection between math and physics is one of the learning goals in many physics courses. The University of Colorado-Boulder, for example, established learning goals for their upper-division quantum mechanics course, one of which is that students should be able to explain the physical meaning of the formal and/or mathematical formulation of a quantum mechanics problem $[5,6]$. The physics education research community has been examining student use of mathematics in a variety of physics courses. For example, Meltzer found a significant correlation between students' mathematics preparation and conceptual learning gains in algebra-based introductory physics courses [7]. Kuo et al. studied how students in a calculus-based introductory physics course blended conceptual and formal mathematical reasoning in solving physics problems [8]. Wilcox et al. applied the ACER framework to analyzing student difficulties in a middle-division classical mechanics course and an upper-division electrostatics course [9]. Bollen et al. found that many students in a second year electrodynamics course struggle with interpreting graphical representations of vector fields and applying vector calculus to physical situations [10].

Previous studies on student understanding of quantum mechanics have tended to center on conceptual understanding [1-4,11-17]. Recently, there is a growing effort to 
research how students make sense of mathematical expressions in quantum mechanics [18,19]. However, little research has specifically focused on students' ability to relate the formalism to real-world quantum phenomena. Zhu and Singh [20] investigated student ability to relate formalism and experimental outcomes in the context of the Stern-Gerlach experiment. In particular, they examined student ability to predict the experimental results when given the spin states. They also evaluated student ability to design experiments to prepare spin states and to distinguish between superposition states and mixed states. The results showed that many students confuse Hilbert space with physical space and have difficulty reasoning about what components of spin need to be measured. Passante et al. [21] probed whether or not students recognize that superposition states and mixed states are different experimentally, as well as the reasoning that students used to justify their answers. They found that many students do not recognize that superposition states and mixed states are experimentally distinguishable. Sayer et al. [22] identified student difficulties with the double-slit experiment. Some of the tasks they used prompted students to write expressions for the number density of particles on the screen under various conditions. They found that many students have difficulty recognizing the effect of photon wavelength and the effect of lamp intensity on the interference pattern. These three studies all reported positive results from assessments of inquiry-oriented curricula, which were developed based on the research findings. We only found one study that directly investigated how students relate patterns of experimental data and the coefficients in a superposition state. Michelini and Zuccarini found that when students are asked to find the coefficients for spin states based on experimental results, most use real numbers and neglect the phases [23]. None of the studies mentioned above, however, explicitly examined the extent to which students are able to relate relative phase and experimental results.

This investigation has two main objectives. The first is to examine the extent to which students recognize that quantum states that differ only by a relative phase can be experimentally distinguished. We conduct an observational experiment, in which students are asked whether or not the quantum states are experimentally distinguishable. We closely examine what specific quantum concepts and mathematical skills are required for relating relative phase and experimental results, as well as student reasoning patterns in relation to what concepts and skills that students have and have not mastered. The second objective is to gain insight into reason(s) why students encounter difficulty in relating relative phase and experimental results. We develop three different hypotheses and conduct experiments using new tasks that require only subsets of the concepts and/or skills necessary for the task used in the observational experiment. We then compare the results to our predictions. Lastly, we synthesize findings from the observational and testing experiments.

This research has been conducted in sophomore- and junior-level quantum mechanics courses at the University of Washington (UW) over a few years. All data presented in this paper have been collected from online surveys. The total number of student participants is 309 .

This paper has five major sections. In Sec. II, we discuss the methods, including research framework, instructional context, data collection, and limitations. In Sec. III, we present results from the observational experiment. Section IV is comprised of results from three testing experiments. This paper concludes with Sec. V, in which we synthesize findings and discuss implications.

\section{METHODS}

\section{A. Research framework}

The perspective on learning we draw on is Heron's pragmatic constructivism [24]. Using this perspective, we study student learning that happens in authentic classroom environments. We assume that learners construct knowledge for themselves. Learners' prior knowledge serves as both a foundation and building material for constructing new knowledge. However, we do not assume that learners' prior ideas are elaborated or stable. Rather, these ideas are considered to depend on the physical context as well as the framing of the problem that students are considering. With this perspective on student learning, we design tasks that focus on certain concepts and mathematical skills but that vary in context or wording to elicit student ideas and reasoning.

The research framework used for this study was adapted from the empirical investigation framework $[24,25]$ from the Physics Education Group at UW. Our investigation is comprised of two different types of experiments: observational experiments and testing experiments. The initial step of the investigation is to conduct an observational experiment, which allows us to elicit student ideas and reasoning, as well as to identify patterns of responses. In an observational experiment, we first design a written task consisting of open-ended questions in which we ask students to explain their reasoning. We then analyze the students' answers and explanations. For student explanations, we use thematic analysis to identify key phrases, generate codes, and characterize patterns of student reasoning [26]. Lastly, we interpret the results and formulate hypotheses to account for the patterns of student reasoning that emerge from the data.

The next step is to conduct testing experiments. To test a hypothesis, we modify the original task to create one or more new tasks, and we make predictions about student performance on the new task(s). We then analyze the new responses and examine whether or not the results are 
consistent with our predictions. If they are consistent, we accept the hypothesis; if not, we reject the hypothesis. Finally, we synthesize findings from the testing experiments. The ultimate goal is to develop instructional activities that make use of the hypotheses to target some of the most prevalent incorrect lines of reasoning.

The statistical tests used throughout this paper are Fisher's exact test [27] and McNemar's test [28]. We chose these tests because our data are categorical (e.g., correct or incorrect), and our samples are small. Fisher's exact is used to help us decide whether or not to aggregate data collected from different student cohorts. McNemar's test is used to compare performance on different tasks that were completed by the same student cohort.

\section{B. Context for research}

This research was conducted in sophomore- and juniorlevel quantum mechanics courses at the UW. We did not collect demographic information from the research participants. Instead, we report demographic data for all students enrolled at the university. Approximately $54 \%$ of all enrolled students (both undergraduate and graduate) are female [29]. The most prevalent races and ethnicities are Caucasian (43\%), Asian (25\%), and international students (16\%) [29]. Below we discuss the structures of these courses and the instructions students receive on relative phase.

\section{Course structures}

The sophomore-level course uses a spins-first approach that covers the first five chapters (and the first section of the sixth chapter) of McIntyre's Quantum Mechanics: A Paradigms Approach [30]. The class meets in a lecture hall 3 times per week, and each class lasts for $50 \mathrm{~min}$. It makes use of interactive activities, such as clicker questions and lecture tutorials based on Tutorials in Physics: Quantum Mechanics [31]. In addition, a prelecture is assigned before almost every lecture. Typically, the prelectures each involve a series of questions either on material that has been covered in the previous lecture(s) or on content that will be introduced in the upcoming lecture. The prelectures are administered online and students receive participation credit. The course is required for all students who graduate with a major or a minor in physics. The typical enrollment during this study is about 200 students. One of the prerequisites for this course is the first of a twoquarter-sequence of mathematical methods in physics, in which complex numbers are covered.

The junior-level course, which is a two-quarter sequence, uses Griffiths' textbook [32]. The course is comprised of two 80-min lectures and a 50-min small-group section each week. The tutorials [31] are used in the small-group sections, led by graduate student teaching assistants. Almost all the students in the junior-level course had previously completed the sophomore-level course (exceptions apply to transfer students). The first quarter covers the first four chapters of Griffiths' [32] and the second quarter covers the rest of the textbook. The first quarter of the sequence is required for almost everyone who will graduate with a physics degree, while the second quarter is optional and is intended for students who will continue to graduate school. The enrollments for the two quarters during this study were 100-110 and $70-80$, respectively.

The tutorials implemented in the small-group sections have three components: (i) online surveys (also known as tutorial pretests), (ii) in-class worksheets, and (iii) tutorial homework. Students are asked to complete an online survey before they attend the small-group section. The online survey involves a series questions, typically on the same topic as the worksheet. The online survey is intended to help prepare students for what they will consider in class. Students receive participation credit but their responses are not graded for correctness. Students do not receive feedback on their responses. They are expected to gain understanding after they work through the in-class worksheets and the homework.

It is worthwhile to mention that the tutorials provide students opportunities for mathematical sensemaking, which has become a recent focus in the physics education and science education research communities [8,18,33]. Gupta and Elby argue that mathematical sensemaking "involves looking for meaning and coherence within the mathematical formalism itself and between the math and the system it describes, if any" [33]. The tutorials make use of guided-inquiry activities to support students in finding coherence between their interpretations of mathematical formalism and of quantum phenomena.

\section{Instruction on relative phase}

The sophomore-level course introduces relative phase in the context of the Stern-Gerlach experiment. Before students are taught quantum states and probabilities, they are introduced to the Stern-Gerlach experiment. A variety of experimental setups are discussed, including sequential measurements (e.g., measure $S_{z}$, then $S_{x}$, and then $S_{z}$ again) on particles with magnetic moments in random directions. After identifying the patterns from the experimental results, students are then introduced to quantum states, inner products, and probabilities. In particular, the course lectures and textbook explicitly discussed how to use the experimental results to infer the coefficients for the expressions of the $S_{x}$ (and $S_{y}$ ) basis states written in the $S_{z}$ basis. In addition, students encounter relative phase again when they study time dependence of spin states. A preliminary version of a tutorial intended to address student difficulties with relative phase was given toward the end of instruction. Students in the sophomore-level course had not completed the tutorial when the research tasks were administered. In two out of three academic years, the junior-level students had completed the tutorial previously in the 
sophomore-level course. However, students from different academic years had very similar performance (see Secs. III and IV).

In the first quarter of the junior-level course, relative phase is discussed in the context of time dependence of wave functions. Typically, spin is not covered in the juniorlevel course until the end of the first quarter or the beginning of the second quarter, depending on the instructor. For spin angular momentum, the junior-level course does not focus on relative phase but rather addition of angular momentum. In one out of three academic years, the junior-level students had completed a short exercise on relative phase in a preliminary version of a tutorial. Again, students from different academic years had very similar performance (see Secs. III and IV).

\section{Data collection}

All the data presented in this paper were students' responses to tasks given in online surveys. All the tasks included multiple-choice questions for which students were asked to explain their reasoning. The multiple-choice format was used to make the preliminary analysis more straightforward, while the explanations provide insights into how students are reasoning about each task.

This paper presents results from seven written tasks that were given to students in either sophomore- or junior-level quantum mechanics courses at the UW. Table I summarizes the number of research participants $N$ (a fraction of students who answered) and the participation rate for each task. The first column indicates the student cohort (e.g., cohorts $\alpha$ and $\beta$ were junior students enrolled in two different academic years) to which each task was given. We note that cohort $\delta$ had just completed the sophomore-level course by the end of this study, and therefore had not received any of the tasks given in the junior-level course. Cohorts $\alpha$ and $\beta$ had the same lecture instructor, while cohort $\gamma$ had a different instructor. Both instructors had taught the junior-level course before. It has been our experience that student performance on qualitative questions does not vary substantially over academic years [3].
Therefore, we assume that student responses from different academic years were comparable and synthesize findings from different academic years.

As shown in Table I, pairs of tasks (e.g., task $2 \mathrm{a}$ and task 2b) were given to students in the same class (e.g., cohort $\gamma$ ) so that direct comparisons could be made. However, none of the tasks were given in the same online survey, which was intended to reduce the probability that similar tasks would elicit similar lines of reasoning. In order to reduce the retest effect, pairs of tasks were given at least one week apart. We assume any retest effect to be small for several reasons: (i) students take online surveys frequently (about once per week in the junior-level course and about 3 times per week in the sophomore-level course), (ii) each online survey involves interrelated but slightly different topics, and (iii) no direct feedback was given to students.

\section{Limitations}

This research has several limitations in the study design. First, we only use data collected online but do not report interview data, which would have allowed us to analyze student reasoning in more detail. Although we have conducted think-aloud interviews, we do not report results from interviews as the interview protocol was different from the tasks presented in this paper. Second, the quantum tasks include both spin-1/2 and infinite square well contexts. Spin has discrete eigenvalues, while the infinite square well involves (discrete) energy and (continuous) position. Therefore, student reasoning may vary when considering different observables. This paper does not address the potential impact that different contexts have on student reasoning about relative phase. Third, two out of four cohorts were given more than two tasks throughout the two-quarter sequence (cohort $\beta$ had three tasks and cohort $\gamma$ had four tasks). It is possible that some students recognized the similarities between these tasks and had thought about them very carefully. In addition, cohort $\gamma$ completed task $2 b$ at the end of the first quarter, and completed task $2 \mathrm{a}$ at the beginning of the second quarter. There was a three-week

TABLE I. Administration of written tasks. Cohort refers to students who are enrolled in the same class. $N$ indicates the number of research participants.

\begin{tabular}{lllll}
\hline \hline & & Course administered & $N$ & Participation rate out of enrollment \\
\hline Cohorts $\alpha$ and $\beta$ & Task 1 & Junior level, second quarter & 86 & $61 \%$ \\
Cohort $\gamma$ & Task 2a & Junior level, second quarter & 49 & $46 \%$ \\
& Task 2b & Junior level, first quarter & 51 & $65 \%$ \\
Cohorts $\beta$ and $\gamma$ & Task 3a & Junior level, first quarter & 136 & $64 \%$ \\
& Task 3b & Junior level, first quarter & 140 & $65 \%$ \\
Cohort $\delta$ & Task 4a & Sophomore level & 83 & $40 \%$ \\
& Task 4b & Sophomore level & 87 & $42 \%$ \\
\hline \hline
\end{tabular}


break between the two quarters. It is possible that the threeweek break had a negative impact on student performance on task $2 \mathrm{a}$.

The data samples presented in this paper also have limitations. The research participants for each task only included a fraction of the enrollment, ranging from $40 \%$ to $65 \%$. Many students choosing not to participate may be due to a lack of incentive. Although we use matched samples to evaluate each testing experiment and make judgments about each hypothesis, the samples presented in this paper may not be well representative of all students enrolled in each course. It is worthwhile to mention that we reviewed the research participants' final course grades. The participation rate was similar for the top three quartiles and somewhat smaller for the bottom quartile across all tasks [34].

\section{OBSERVATIONAL EXPERIMENT: STUDY 1}

In study 1 , we designed a task to examine the extent to which students recognize the measurable effects of relative phase. We chose the context of spin $1 / 2$ because students are expected to be familiar with the Stern-Gerlach experiment, which demonstrates the relationship between relative phase and probabilities. In addition, relative phase and various setups of the Stern-Gerlach experiment are taught extensively in the sophomore-level quantum mechanics course. The task was inspired by questions used by Passante et al. to probe student ability to distinguish superposition states from mixed states [21].

\section{A. Spin context with experiment prompt}

Task 1 (see Fig. 1) was given to students in the second quarter of the junior-level course, toward the beginning of instruction. It was given to cohorts $\alpha$ and $\beta$ enrolled in two different academic years. Students had been taught spin in both the sophomore- and junior-level courses. On task 1 , students considered two ensembles of spin- $1 / 2$ particles. The particles in each ensemble are represented by a superposition state written in the $S_{z}$ basis. The coefficients have identical magnitudes but the coefficients for the second terms have opposite signs and thus the relative

Task 1

Consider the following two ensembles of spin-1/2 particles: The particles in ensemble $A$ are all described by the state vector $\left|\psi_{A}\right\rangle=\frac{1}{2}|+\rangle_{z}+\frac{\sqrt{3}}{2}|-\rangle_{z}$.

The particles in ensemble $\mathrm{B}$ are all described by the state vector $\left|\psi_{B}\right\rangle=\frac{1}{2}|+\rangle_{z}-\frac{\sqrt{3}}{2}|-\rangle_{z}$.

Is there an experiment that can be used to distinguish the particles in ensemble A from the particles in ensemble B? Explain your reasoning.

A. Yes, there is an experiment.

B. No, there is not an experiment.

C. Not enough information.

FIG. 1. A task with an experiment prompt in spin context used in study 1 . phases between the basis states are different. Students were asked whether or not there is an experiment that can be used to distinguish the particles in ensemble $A$ from the particles in ensemble $B$.

In order to answer whether or not the states can be distinguished, students might recognize that although the probabilities of measuring either $+\hbar / 2$ or $-\hbar / 2$ for $S_{z}$ are identical for the two spin states (since the coefficients in the $z$ basis for each state have identical magnitudes), the results for measuring spin can be different in the $x$ or $y$ direction. The probability of measuring $+\hbar / 2$ for $S_{x}$, for example, can be found by taking the modulus squared of the inner product between the bra ${ }_{x}\langle+|$ and the state vector $|\psi\rangle$ : $\left.\left.\right|_{x}\langle+\mid \psi\rangle\right|^{2}$. Since the given states are written in the $S_{z}$ basis, students need to know how to convert from the $S_{x}$ basis to the $S_{z}$ basis. Hence, the probabilities of measuring either $+\hbar / 2$ or $-\hbar / 2$ for $S_{x}$ are different since the inner products yield values with different magnitudes. In general, the probabilities of measuring either $+\hbar / 2$ or $-\hbar / 2$ along an arbitrary direction are also different. Thus, one can distinguish the particles from ensembles $A$ and $B$ by measuring $S_{x}$, or the spin component along an arbitrary direction.

\section{B. Results}

Table II shows the overall student performance on all tasks, including task 1 . The results are aggregated because the difference in percentages of correct answers between cohorts $\alpha$ and $\beta$ is not statistically significant (Fisher's exact test, $p=0.385)$. Only two students $(2 \%, N=86)$ selected "not enough information" as their answer choice. About half of the students $(47 \%, N=86)$ answered correctly that the ensembles are experimentally distinguishable, and about one-third (35\%, $N=86)$ gave correct explanations. Explanations were considered correct if they included either a discussion of what observable(s) need to be measured $(29 \%, N=86)$ or pointed out that the relative phases are different $(6 \%, N=86)$.

Most of the students $(38 \%, N=86)$ who answered that the ensembles are not experimentally distinguishable

TABLE II. Student performance on tasks concerning relative phase.

\begin{tabular}{lcccc}
\hline \hline & & $N$ & $\begin{array}{c}\text { Correct } \\
\text { answer }\end{array}$ & $\begin{array}{c}\text { Correct } \\
\text { explanation }\end{array}$ \\
\hline Cohorts $\alpha$ and $\beta$ & Task 1 & 86 & $47 \%$ & $35 \%$ \\
Cohort $\gamma$ & Task 2a & 49 & $61 \%$ & $41 \%$ \\
Cohorts $\beta$ and $\gamma$ & Task 2b & 51 & $59 \%$ & $31 \%$ \\
& Task 3a & 136 & $54 \%$ & $15 \%$ \\
Cohort $\delta$ & Task 3b & 140 & $50 \%$ & $18 \%$ \\
& Task 4a & 83 & $94 \%$ & $\ldots$ \\
\hline \hline
\end{tabular}


explained that the probabilities are the same without explicitly specifying the components of spin. For example, one student explained that

"I don't know what it means to have a negative coefficient, but if I'm still taking the square of it to find the probability, then it won't have an effect and the two ensembles will look identical."

Only three students $(3 \%, N=86)$ explicitly stated that the probabilities would be the same if one measures any direction of the spin. Furthermore, four students $(5 \%$, $N=86$ ) explained that the (relative) phase does not affect probabilities.

\section{Formulate hypotheses}

In order to account for student difficulty in relating relative phase and experimental results, we formulated three hypotheses. These hypotheses were formulated based on two aspects: (i) physics experts' views of what specific quantum concepts and mathematical skills are required and (ii) student reasoning patterns in relation to what concepts and skills that students have and have not mastered. We recognized that task 1 was sophisticated as students were required not only to recognize what spin component(s) might be measured, but also to be fluent with determining probability as well as to be proficient with complex numbers. Thus, we intended to unpack the task and to have each hypothesis address one element of the requirement. As discussed above, many students explained that the quantum states are not experimentally distinguishable because the probabilities are the same, and yet very few explicitly specified which observables they thought have the same probabilities. Since the quantum states were expressed in the $S_{z}$ basis, it is possible that many students were only considering the probabilities for the $z$ component of spin. Therefore, one of the hypotheses was intended to address student reasoning concerning the concepts of quantum measurement and probability. The other two hypotheses were intended to address student fluency with the mathematical formalism for probability, and proficiency

Task 2a

Consider the following two ensembles of spin-1/2 particles: The particles in ensemble $\mathrm{A}$ are all described by the state vector $\left|\psi_{A}\right\rangle=\frac{1}{2}|+\rangle_{z}+\frac{\sqrt{3}}{2}|-\rangle_{z}$

The particles in ensemble $B$ are all described by the state vector $\left|\psi_{B}\right\rangle=\frac{1}{2}|+\rangle_{z}-\frac{\sqrt{3}}{2}|-\rangle_{z}$.

Will the probability that ensemble $\mathrm{A}$ is measured to have spin up in the $x$-direction be the same as or different from the probability that ensemble B is measured to have spin up in the $x$-direction? Explain your reasoning.
A. Same as
B. Different from
C. Not enough information

with complex number, respectively. These two hypotheses also needed to be tested because regardless of what probabilities students considered, lacking skill with the formalism for probability or with complex numbers may still have hindered student ability to recognize the measurable effects of relative phase. All three hypotheses are stated below:

Hypothesis A: Prompting probabilities for a specific observable improves student ability to recognize the measurable effects of relative phase.

Hypothesis B: Prompting probabilities in a case that does not require taking inner products (or changing basis) improves student ability to recognize the measurable effects of relative phase.

Hypothesis $C$ : Lacking proficiency with distinguishing the modulus squared of complex numbers has hindered student ability to recognize the measurable effects of relative phase.

\section{TESTING EXPERIMENTS}

In this section, we describe the tasks designed for testing the hypotheses. Each hypothesis was tested in one of the studies. We also report results from each testing experiment.

\section{A. Study 2: Testing hypothesis $A$}

In order to test hypothesis $A$ (prompting probabilities for a specific observable improves student ability to recognize the measurable effects of relative phase), two different tasks (see Fig. 2) were used. Task 2a specifically prompts students to compare the probabilities for a specific component of spin. Task $2 \mathrm{~b}$, which is identical to task 1 except for the coefficients, was used for comparison of student performance. Task $2 \mathrm{~b}$ was given toward the end of the first quarter of the junior-level course, after students received lecture instruction on spin. Task $2 \mathrm{a}$ was then administered in the first week of the subsequent quarter, after a threeweek break. We note that students did not receive instruction or feedback on the concept of relative phase between the two tasks. Therefore, we assume no significant retest effect. We predicted that student performance on task 2a

\section{Task 2b}

Consider the following two ensembles of spin-1/2 particles:

The particles in ensemble $\mathrm{A}$ are all described by the state vector $\left|\psi_{A}\right\rangle=\frac{3}{5}|+\rangle_{z}+\frac{4}{5}|-\rangle_{z}$.

The particles in ensemble $B$ are all described by the state vector $\left|\psi_{B}\right\rangle=\frac{3}{5}|+\rangle_{z}+\frac{4}{5} e^{i \pi / 2}|-\rangle_{z}$.

Is there an experiment that can be used to distinguish the particles in ensemble A from the particles in ensemble B? Explain your reasoning.

A. Yes, there is an experiment.

B. No, there is not an experiment.

C. Not enough information.

FIG. 2. A task with a probability prompt and a task with an experiment prompt in spin context used in study 2 . 
(probability prompt) would be substantially better than on task $2 \mathrm{~b}$ (experiment prompt) if hypothesis $A$ is correct. Below we describe task $2 \mathrm{a}$ and report the results on both tasks $2 \mathrm{a}$ and $2 \mathrm{~b}$.

\section{Spin context with probability prompt}

The scenario of task $2 \mathrm{a}$ is the same as task 1 , in which students consider two ensembles of spin- $1 / 2$ particles. However, the prompt in task $2 \mathrm{a}$ is different: students are instead asked to compare the probabilities of measuring $S_{x}$ for particles in the two ensembles. As discussed in Sec. III A, the probabilities of measuring $S_{x}$ are different due to the different relative phases.

\section{Results}

The overall student performance on task $2 \mathrm{a}$ is shown in Table II. About $61 \%$ answered correctly that the probabilities for $S_{x}$ are different, and $41 \%$ gave correct explanations. Student explanations were considered correct if they stated that after taking an inner product (or changing basis), the different signs or phases in the other term would result in different probabilities. The values for the probabilities were not required as part of a correct explanation.

Student performance on task $2 \mathrm{~b}$ is also shown in Table II. On both task $2 \mathrm{a}$ and task $2 \mathrm{~b}$, the percentage of correct answers as well as the percentage of correct explanations are similar. Very few students chose "not enough information" ( 2 students on task $2 \mathrm{a}$ and 3 students on task $3 b$ ). In addition, the results from tasks $2 \mathrm{a}$ and $2 \mathrm{~b}$ are similar to results from task 1.

In order to compare how individual students answered the tasks with different prompts, we eliminated responses from students who did not answer one of the tasks. We only compared student answers but not explanations, as correct explanations for different tasks had different criteria, which make them difficult to compare. Table III shows the number of students who gave correct and/or incorrect answers on tasks with different prompts. McNemar's test result $(p=0.752)$ suggests that the difference in percent correct is not statistically significant.

We then examined the explanations given by students who incorrectly answered that the probabilities for $S_{x}$ are the same on task 2a. Ten out of seventeen students explained that the (modulus) squared of the coefficients

TABLE III. Response patterns for students who answered both task $2 \mathrm{a}$ and task $2 \mathrm{~b}(N=27)$.

\begin{tabular}{lcc}
\hline \hline & \multicolumn{2}{c}{ Task 2b (experiment prompt) } \\
\hline Task 2a (probability prompt) & Correct & Incorrect \\
\hline Correct & 10 & 6 \\
Incorrect & 4 & 7 \\
\hline \hline
\end{tabular}

are the same and another four students explained that the coefficients (or the results of inner products) are the same. None of these students showed the steps of the calculations in their responses, which may be due to the fact that the task was administered online.

\section{Reflection on results}

The results suggest that performance does not improve when students are prompted to compare probabilities for $S_{x}$. Therefore, we reject hypothesis $A$. Although the results do not allow us to conclude whether or not students who answered the experiment prompt version incorrectly indeed only considered the probabilities for $S_{z}$, it does suggest that other factors impacted student performance. In order to answer the specific prompt version correctly, two steps of computation are needed: (i) take an inner product such as ${ }_{x}\langle+\mid \psi\rangle$ or rewrite the state in the $S_{x}$ basis, and (ii) take the modulus squared of the inner product or of the coefficient for $|+\rangle_{x}$. Students who answered incorrectly did not show evidence of computation. Thus, it was difficult to pinpoint where students made errors. Studies 3 and 4 evaluate the extent to which lacking fluency with taking inner products and lacking proficiency with complex numbers may have hindered student ability to recognize the measurable effects of relative phase. We note it is not possible to determine if students performed a computation on paper before answering, if they performed a mental computation, or if they performed no computation at all. We take this into account for the design of studies 3 and 4 .

\section{B. Study 3: Testing hypothesis $B$}

In study 3 , we tested hypothesis $B$ : prompting probabilities in a case that does not require taking inner products (or changing basis) improves student ability to recognize the measurable effects of relative phase. Considering the possible inconvenience of performing computation online, we decided to design a task that does not require taking inner products (or changing basis). We chose the infinite square well context because in this context, a positionspace wave function is typically used and can be written as a linear combination of energy eigenfunctions. Wave functions with different relative phases between energy eigenfunctions can be used so that students do not need to take inner products or change basis explicitly, as was required in the spin context. Below we describe the two tasks used in study 3 .

\section{Infinite square well context}

Two infinite square well context tasks are shown in Fig. 3. Task $3 \mathrm{a}$ is analogous to task $2 \mathrm{a}$ with prompts for specific probabilities, and task $3 \mathrm{~b}$ is analogous to task $2 \mathrm{~b}$ (and task 1) with experiment prompts. Task $3 \mathrm{a}$ and task $3 \mathrm{~b}$ were given to cohorts $\beta$ and $\gamma$ in the first quarter of the junior-level course. We note that students had completed an 
Task 3a

Consider the two wave functions given below for particles $\mathrm{A}$ and B, each in an infinite square well (where $N$ is a constant):

$\psi_{A}(x)=N\left(\varphi_{1}(x)+\varphi_{3}(x)\right)$

$\psi_{B}(x)=N\left(\varphi_{1}(x)+i \varphi_{3}(x)\right)$

Recall that the energy eigenfunctions for the infinite square well are given by: $\varphi_{n}(x)=\sqrt{\frac{2}{a}} \sin \left(\frac{n \pi x}{a}\right)$

Would the position-space probability densities for these two particles be the same or different? Explain your reasoning.

A. Same

B. Different

C. Not enough information
Task 3b

Consider two ensembles of identical particles in infinite square wells. There is one particle in each well. The particles in ensemble $\mathrm{A}$ are all described by the wave function $\psi_{A}(x)=\frac{1}{\sqrt{2}} \varphi_{1}(x)+\frac{1}{\sqrt{2}} \varphi_{2}(x)$.

The particles in ensembles $\mathrm{B}$ are all described by the wave function $\psi_{B}(x)=\frac{1}{\sqrt{2}} \varphi_{1}(x)+e^{i \pi / 2} \frac{1}{\sqrt{2}} \varphi_{2}(x)$, where $\varphi_{1}(x)$

and $\varphi_{2}(x)$ are the eigenfunctions for the ground state and first-excited state.

Is there an experiment that can be used to distinguish the particles in ensemble A from particles in ensemble B? Explain your reasoning.

A. Yes, they are experimentally distinguishable.

B. No, they are not experimentally distinguishable.

C. Not enough information.

FIG. 3. A task with a probability density prompt and a task with an experiment prompt in the infinite square well context used in study 3 .

interactive lecture tutorial at the end of the sophomorelevel course, which was shown to be effective at helping students recognize that superposition states and mixed states are experimentally distinguishable [21]. The tutorial involved a scenario in which students considered position measurements in the infinite square well context. Therefore, we assumed that the students were familiar with considering experiments in the infinite square well context to some extent.

In one academic year, task $3 \mathrm{a}$ was given one week before task $3 \mathrm{~b}$ was given, while in the other academic year, task $3 \mathrm{a}$ was given two weeks before task $3 b$. Another difference in conditions was that in one of the academic years, students' only lecture instruction on the infinite square well was in the prerequisite sophomore-level course. However, in both academic years, students had been introduced to positionspace probability and probability density. We also note that students from both academic years worked through a problem related to relative phase from a preliminary version of tutorial homework before taking task $3 \mathrm{~b}$ but after task $3 \mathrm{a}$. However, we assumed that the impact on student performance on task $3 b$ was not significant for two reasons: (i) the homework problem did not resemble the research tasks since it used graphical position-space wave functions (instead of algebraic expressions) and asked for energy probability (instead of position probability), and (ii) students received feedback on the homework problem in only one of the academic years and yet the results from the two academic years are essentially the same, as will be discussed below.

Probability density prompt: On task $3 \mathrm{a}$, students are given the wave functions for two different particles. Similar to task $2 \mathrm{a}$, they are asked to compare the position-space probability densities for these two particles. Since the relative phase between the energy eigenfunctions are different for these particles, the position-space probability densities are different: one is $N^{2}\left[\varphi_{1}^{2}(x)+\varphi_{3}^{2}(x)+\right.$ $\left.2 \varphi_{1}(x) \varphi_{3}(x)\right]$ and the other one is $N^{2}\left[\varphi_{1}^{2}(x)+\varphi_{3}^{2}(x)\right]$.

Experiment prompt: On task $3 b$, students consider two ensembles of identical particles in infinite square wells. Similar to task $2 \mathrm{~b}$ (and task 1), they are asked whether or not the particles in ensembles $A$ and $B$ are experimentally distinguishable. The probability density for particles in ensemble $A$ can be written as $\frac{1}{2}\left[\varphi_{1}^{2}(x)+\varphi_{2}^{2}(x)+\right.$ $\left.2 \varphi_{1}(x) \varphi_{2}(x)\right]$ and the probability density for particles in ensemble $B$ as $\frac{1}{2}\left[\varphi_{1}^{2}(x)+\varphi_{2}^{2}(x)\right]$. Hence, the probability density for the particles in ensemble $B$ is symmetric about the midpoint of the well, whereas for ensemble $A$ it is asymmetric, with higher probability on the left. Thus, one can distinguish the particles from those two ensembles by measuring position.

\section{Results}

Table II shows the aggregated data for each task used in study 3 . There is no significant difference between cohorts $\beta$ and $\gamma$ (Fisher's exact test, $p=0.311$ ). On both tasks, only three students chose "not enough information."

On task 3a, approximately $54 \%(N=136)$ of the students gave correct answers, and 15\% $(N=136)$ provided correct explanations. Students' explanations were considered correct if they either gave the correct expressions for the probability densities or stated that the probability density for particle $\mathrm{B}$ does not have the cross term. Approximately $24 \%(N=136)$ of the students explained that the imaginary number would make a difference in the probability density but did not provide justification. These explanations were not considered correct. In addition, about $7 \%(N=136)$ of the students incorrectly explained that the complex number $i$ would result in a negative term in the expression of the probability density for particle $B$.

On task 3b, approximately 50\% $(N=140)$ of the students gave correct answers, and 18\% $(N=140)$ gave correct explanations. Students' explanations were considered correct if they either stated that one can measure position (or the probability densities are different), or the relative phases are different (or measure a different basis). About $6 \%(N=140)$ incorrectly explained that SternGerlach experiments can be used to distinguish the 
TABLE IV. Response patterns for students who answered both task $3 \mathrm{a}$ and task $3 \mathrm{~b}(N=126)$.

\begin{tabular}{lcc}
\hline \hline & \multicolumn{2}{c}{ Task 3b (experiment prompt) } \\
\cline { 2 - 3 } Task 3a (probability & Correct & Incorrect \\
\hline density prompt) & 40 & 28 \\
Correct & 22 & 36 \\
\hline Incorrect & & \\
\hline \hline
\end{tabular}

particles. In addition, another $4 \%(N=140)$ explained that one can measure the particles along the $y$ or $z$ axis. These students appeared to recognize this task as similar to what they learned from Stern-Gerlach experiments, but incorrectly generalized the idea to the new context.

To examine how each student answered similarly or differently on each task, students' answers to task 3a and task $3 \mathrm{~b}$ were matched. Table IV shows the numbers of students who gave correct and/or incorrect answers on tasks $3 \mathrm{a}$ and $3 \mathrm{~b}$. McNemar's test result $(p=0.480)$ suggests that there is no statistically significant difference in the percent correct between tasks $3 \mathrm{a}$ and $3 \mathrm{~b}$. We only compare student answers because correct explanations for tasks $3 \mathrm{a}$ and $3 \mathrm{~b}$ had different criteria. A correct explanation for task $3 b$ required stating how the probability densities are different, while a correct explanation for task $3 a$ only required stating that one can measure position (or probability densities are different).

We examined explanations given by students who answered incorrectly on either task $3 a$ or $3 b$. On the probability density prompt version (task $3 \mathrm{a}$ ), the most common $(31 \%, N=136)$ explanation was that the imaginary number does not affect the probability density. Many (23\%, $N=136)$ of these students further explained that the imaginary number would be "canceled" when taking the modulus squared. Similarly, on the experiment prompt version (task 3b), approximately $27 \%(N=140)$ explained that the probabilities or probability densities are the same, and another $14 \%(N=140)$ explained that the (relative) phase does not affect probability. For example, a student explained that

\section{"Experiments can only determine the probability of the states but cannot determine the phase information of the states. In other words, the phase information is described by imaginary part and that cannot be determined experimentally. Particles in A and B both has possibility of $1 / 2$ in ground state and first- excited state, so the experimental result should be the same."}

This student recognized that the experimental outcomes would provide information about the probability distributions, but did not think the phase can be determined experimentally because it is imaginary.

\section{Reflection on results}

The results suggest that performance does not improve when students are prompted to compare probabilities in a case that does not require taking an inner product (or changing basis). Therefore, we reject hypothesis $B$. We note that the results do not allow us to conclude whether or not students who answered task 1 incorrectly made errors in taking inner products. However, when specifically prompted to compare position-space probability densities, many still explained that the imaginary number does not affect the probability density. This suggests a need for examining the extent to which proficiency with complex number has impacted student ability to recognize the measurable effects of relative phase.

\section{Study 4: Testing hypothesis $C$}

In study 4 , two tasks were used to test hypothesis $C$. One was in a purely mathematical context (task 4a) and the other was in a spin- $1 / 2$ context with an experiment prompt (task 4b). Both tasks were administered in the sophomorelevel quantum mechanics course. Task $4 \mathrm{a}$ was administered at the beginning of the academic quarter, before quantum probability were introduced. Task $4 \mathrm{~b}$ (experiment prompt in spin context) was then given to students after lecture instruction on relative phase and probability.

Results from study 3 suggest that many students appear to think (incorrectly) that the modulus squared of the wave functions are the same. Therefore, we administered task $4 \mathrm{a}$ at the beginning of the sophomore-level course in order to eliminate the impact of student reasoning with quantum probability on how students answer in a purely math context. This allowed us to examine whether or not students were proficient with comparing the modulus squared of complex numbers. We assumed that few, if any, of the students had studied quantum mechanics before taking the sophomore-level course, and therefore reasoning about quantum probabilities would not interfere with students' reasoning in the math context. If students lack mathematical proficiency with finding the modulus squared of complex numbers, student performance on task $4 \mathrm{a}$ would be about the same or even worse than on task $4 \mathrm{~b}$.

\section{Purely mathematical context}

As shown in Fig. 4, task 4a involves a purely mathematical context. Students consider two complex numbers $\left(\frac{3}{5}+\frac{4}{5}\right.$ and $\left.\frac{3}{5}+i \frac{4}{5}\right)$ and they are asked whether the modulus squared of these complex numbers are the same or different. The correct answer is that they are different (49/25 vs 1$)$.

Task $4 \mathrm{~b}$ is almost identical to task 1 and task $2 \mathrm{~b}$, except for the coefficients. However, the coefficients in task $4 \mathrm{~b}$ are the same as the components of the complex numbers in task $4 \mathrm{a}$, which allows us to make a direct comparison of student performance in the different contexts. 
Task 4a

Consider the complex numbers below:
$\frac{3}{5}+\frac{4}{5}$
$\frac{3}{5}+i \frac{4}{5}$
Would $\left|\frac{3}{5}+\frac{4}{5}\right|^{2}$ be the same as or different from $\left|\frac{3}{5}+i \frac{4}{5}\right|^{2}$ ?
Explain your reasoning.
A. Same as
B. Different from
C. Not enough information

Task 4b

Consider the following two ensembles of spin-1/2 particles: The particles in ensemble $\mathrm{A}$ are all described by the state vector $\left|\psi_{A}\right\rangle=\frac{3}{5}|+\rangle_{z}+\frac{4}{5}|-\rangle_{z}$.

The particles in ensemble $\mathrm{B}$ are all described by the state vector $\left|\psi_{B}\right\rangle=\frac{3}{5}|+\rangle_{z}+i \frac{4}{5}|-\rangle_{z}$.

Is there an experiment that can be used to distinguish the particles in ensemble A from the particles in ensemble B? Explain your reasoning.

A. Yes, there is an experiment.

B. No, there is not an experiment.

C. Not enough information.

FIG. 4. A task in purely mathematical context and a task with an experiment prompt in spin context used in study 4.

\section{Results}

Sophomore-level students' performance on task 4a and $4 \mathrm{~b}$ is shown in Table II. Most students (94\%, $N=83$ ) correctly recognized the modulus squared of the two complex numbers are different on task $4 \mathrm{a}$. We did not determine the percentage of correct answers with correct explanations because the extent to which students explained in detail varied. For students who gave correct answers, 54\% $(N=83)$ provided results from computations, and the rest $(40 \%, N=83)$ explained qualitatively. About $35 \%(N=83)$ gave correct numerical results for both modulus squared, and $17 \%$ made errors in computing $\left|\frac{3}{5}+i \frac{4}{5}\right|^{2}$. The most salient error appears to be treating modulus squared (e.g., $\left|\frac{3}{5}+i \frac{4}{5}\right|^{2}$ ) as squared [e.g., $\left(\frac{3}{5}+i \frac{4}{5}\right)^{2}$, which has been documented in prior research [35-37].

On task $4 \mathrm{~b}$, in contrast, only $66 \%(N=87)$ correctly answered that the spin states are experimentally distinguishable, and 38\% $(N=87)$ gave correct explanations. The high percentage (above 90\%) of correct answers on task 4a suggests that most of the students are proficient with distinguishing the modulus squared of complex numbers. However, in order to eliminate the possibility that the participants on both tasks come from different subsets of students, we only examined responses from students who answered both tasks. Table V shows the numbers of students who gave correct and/or incorrect answers on tasks $4 \mathrm{a}$ and $4 \mathrm{~b}$. Again, we use McNemar's test and the result $(p<0.001$, odds ratio 12.5) suggests that students performed significantly better on the mathematical context than on the spin context.

TABLE V. Response patterns for students who answered both task $4 \mathrm{a}$ and task $4 \mathrm{~b}(N=82)$.

\begin{tabular}{lcc}
\hline \hline & \multicolumn{2}{c}{ Task 4b (spin experiment prompt) } \\
\cline { 2 - 3 } Task 4a (math context) & Correct & Incorrect \\
\hline Correct & 52 & 25 \\
Incorrect & 2 & 3 \\
\hline \hline
\end{tabular}

\section{Reflection on results}

The results of study 4 suggest that sophomore-level students performed substantially better in the purely mathematical context than in spin context with an experiment prompt. Although errors were made when computing the modulus squared of the complex number with nonzero real and imaginary parts, most of the sophomore-level students were able to distinguish $\left|\frac{3}{5}+\frac{4}{5}\right|^{2}$ and $\left|\frac{3}{5}+i \frac{4}{5}\right|^{2}$. Therefore, we reject hypothesis $C$ that lacking proficiency with distinguishing the modulus squared of complex numbers has hindered student ability to recognize the measurable effects of relative phase. We believe that this conclusion is reasonable not only for sophomore-level students, but for junior-level students as well. This conclusion was made based on an assumption that students' mathematical skills remain steady or improve throughout their progression in sophomore- and junior-level courses.

\section{DISCUSSION AND CONCLUSION}

This paper presents an investigation into student reasoning in relating quantum formalism and real-world phenomena. Specifically, the concept of relative phase was chosen as an example. In order to evaluate the extent to which students are able to recognize the measurable effects of relative phase as well as to gain insights into reason(s) why students have difficulty in relating relative phase and experimental results, one observational experiment (in study 1) and three testing experiments (in studies 2-4) were conducted.

\section{A. Synthesis of findings}

Study 1 showed that about half of the junior-level students did not recognize that particles with different relative phases in spin states are experimentally distinguishable. The task used in study 1 was sophisticated as students not only needed to recognize what spin component(s) might be measured, but also needed to be fluent with the formalism for determining probabilities as well as be proficient with complex numbers. We then unpacked these elements in studies 2-4 through testing hypotheses. The formulation of hypotheses was informed by both the 
knowledge of what concepts and skills are required for the tasks and the patterns of student responses and explanations. The goal was to evaluate the extent to which each concept or skill may have hindered students' ability to recognize the measurable effects of relative phase.

In studies 2 and 3, we designed tasks that eliminated some of the steps required for the original task (with an experiment prompt) in study 1 . On one task in study 2 , students did not need to consider what spin component to measure; similarly, on one task in study 3 , students did not need to either (i) consider what observable to measure or (ii) take an inner product (or change basis). In both studies, we compared student performance on the new task and a variant to the original task. Students' similar performance led us to reject both hypothesis $A$ (prompting probabilities for a specific observable improves student ability to recognize the measurable effects of relative phase) and hypothesis $B$ (prompting probabilities in a case that does not require taking inner products improves student ability to recognize the measurable effects of relative phase).

In study 4 , we investigated the extent to which lacking proficiency with complex numbers may have hindered student ability to recognize the measurable effects. We administered a task in which students were prompted to compare the modulus squared of two complex numbers in the sophomore-level course before students were introduced to quantum probabilities. For comparison, students were also given an experiment task (in a spin-1/2 context) after they received instruction on relevant topics. The results showed that sophomore-level students performed significantly better in the math context. We assume that junior-level students have higher or equivalent mathematical skill compared to sophomore-level students. Thus, we rejected hypothesis $C$ and concluded that proficiency with complex numbers is not a barrier to successfully recognizing the measurable effects of relative phase.

\section{B. Many students do not recognize the purpose of using complex coefficients in superposition states}

Our results suggest to us that many sophomore- and junior-level students do not recognize the purpose of using complex coefficients in superposition states. This claim is supported by our finding that students are proficient with distinguishing the modulus squared of complex numbers, and yet they often do not apply this skill correctly when comparing quantum probabilities. As a result, students often fail to recognize the measurable effects of different relative phases.

Our results align with Loverude's findings from studying students' use of mathematics in physics. Loverude argues that "experience with mathematical procedures does not necessarily mean students make sense of the meaning of the procedures" [38]. Indeed, our students, who had completed one term of mathematical methods, were proficient at distinguishing modulus squared of complex numbers, but often failed to make sense of the analogous comparison in quantum mechanics. As discussed previously, students tended to state "I don't know what it means to have a negative coefficient," or "the phase cannot be determined experimentally."

Loverude also claims that "models of learning transfer suggest strongly that students need to learn math in context." Our empirical results support this argument: our students did not spontaneously transfer mathematical proficiency to quantum contexts. Regardless of whether the task prompted for an experiment or for a specific probability, students commonly stated that the probabilities are the same because the probability is calculated by a modulus squared. Although we do not have direct evidence about whether or not students recognize that the quantum tasks are analogous to the purely mathematical task, students' substantially better performance on the purely mathematical task suggests a gap between students' mathematical skill and their sensemaking of math in physics.

\section{Possible explanations for why students fail to recognize the purpose of using complex coefficients in superposition states}

Our data show that regardless of whether the task is in the spin-1/2 or the infinite square well context, and whether it has an experiment prompt or a probability prompt, students tend not to recognize the measurable effects of relative phase. Students commonly justify their answers by stating that the modulus squared of probability amplitudes that include different relative phases are the same. When we examined student responses in greater detail, we found that some appear to reflect students' interpretation of the role that complex numbers play in quantum states. For example, one student explained that

"because the complex term in the B particle will cancel out in measuring a probability, there is no experimental way to distinguish the two particle systems." (task 3b)

This student appeared to focus on the fact that a complex number will be canceled out after multiplied by its complex conjugate, and therefore did not think the ensembles are experimentally distinguishable. Similarly, another student explained that

"There [sic] difference is a phase, which cannot be measured because it is imaginary." (task 3b)

The belief that imaginary coefficients cannot be measured may be due to the fact that the eigenvalues of physical observables and their probabilities are real, as well as that classical mechanics only makes use of real numbers. Therefore, students struggle to reconcile the measurable effects of relative phase with physical observables that have real eigenvalues. 
The belief about "imaginary things cannot be measured" does not only arise in the tasks used in this study. Research on student understanding of time dependence and measurements also reported similar ideas [1,2]. Passante et al. [2], for example, found that some students think that an imaginary coefficient in front of an energy eigenstate means that the corresponding energy is not measurable. Emigh et al. [1] reported that although some students correctly wrote down the time-dependent wave function for a superposition of energy eigenstates, they incorrectly concluded that the probability density does not depend on time because "probability is squared and [the] time component won't matter."

\section{Implications for future research and instruction}

Similar patterns about students' interpretation of the role that complex numbers play in quantum mechanics emerged in a variety of tasks. Although the findings mentioned above all emerge from empirical investigations, these patterns can be explained from theoretical perspectives as well. For example, Passante [39] has begun to analyze student responses to quantum mechanics tasks through the lens of conceptual resources [40]. She identified "energy cannot be imaginary" as a resource element activated by some students when prompted to determine possible value(s) for an energy measurement. Here, we identify a different theoretical framework that may be productive to guide a further empirical investigation: dual process theories of reasoning [41,42]. The data presented in this paper suggest that while students appear to be familiar with the formalism for determining quantum probabilities and are proficient with complex numbers, many still tend to rely on an incorrect but intuitively appealing idea that "phase cannot be measured." This suggests that the quantum tasks used in this study may elicit an intuitively appealing but incorrect response. Dual process theories have been increasingly helpful for examining students' intuitive thinking in introductory physics $[42,43]$, but we are not aware of any studies that apply a dual-process theory in upper-division quantum mechanics. We suggest the development and investigation of such a dual-process theory to help account for students' intuitive thinking in quantum mechanics as a line of future research.

Findings from this and many other investigations together suggest that students do not spontaneously transfer their mathematical skills to physics contexts. Students who are fluent with mathematical procedures do not necessarily make sense of the mathematics in quantum mechanics, by which we mean that students often do not consider the meaning, purpose, and implications of mathematical elements of physical theories (such as the use of complex numbers in quantum mechanics). We suggest that instructors should provide opportunities and encourage students to make sense of math in the context of quantum mechanics rather than expecting students to apply their mathematical skills spontaneously to quantum tasks. To help students relate relative phase and experimental results, we suggest curriculum developers design questions that elicit students' intuitions about the role that complex numbers play in quantum mechanics, and then guide students to find coherence between the experimental results and their intuitions.

\section{ACKNOWLEDGMENTS}

The authors would like to thank Amy Robertson for her insightful comments on this study. We also thank Sheh Lit Chang for helping collect data. This work was supported in part by National Science Foundation Grants No. 1022449 and No. 1821032.
[1] P. J. Emigh, G. Passante, and P. S. Shaffer, Student understanding of time dependence in quantum mechanics, Phys. Rev. ST Phys. Educ. Res. 11, 020112 (2015).

[2] G. Passante, P. J. Emigh, and P. S. Shaffer, Examining student ideas about energy measurements on quantum states across undergraduate and graduate levels, Phys. Rev. ST Phys. Educ. Res. 11, 020111 (2015).

[3] P. J. Emigh, G. Passante, and P. S. Shaffer, Developing and assessing tutorials for quantum mechanics: Time dependence and measurements, Phys. Rev. Phys. Educ. Res. 14, 020128 (2018).

[4] G. Zhu and C. Singh, Surveying students' understanding of quantum mechanics in one spatial dimension, Am. J. Phys. 80, 252 (2012).

[5] S. Goldhaber, S. J. Pollock, M. Dubson, P. Beale, and K. K. Perkins, Transforming Upper-Division Quantum
Mechanics: Learning Goals and Assessment, in Proceedings of the 2009 Physics Education Research Conference, Ann Arbor, Michigan (AIP, New York, 2009).

[6] https://physicscourses.colorado.edu/EducationIssues/ Quantum/learning_goals.html. Retrieved 1/15/2019.

[7] D. E. Meltzer, The relationship between mathematics preparation and conceptual learning gains in physics: A possible "hidden variable" in diagnostic pretest scores, Am. J. Phys. 70, 1259 (2002).

[8] E. Kuo, M. M. Hull, A. Gupta, and A. Elby, How students blend conceptual and formal mathematical reasoning in solving physics problem, Sci. Educ. 97, 32 (2013).

[9] B. R. Wilcox, M. D. Caballero, D. A. Rehn, and S. J. Pollock, Analytic framework for students' use of mathematics in upper-division physics, Phys. Rev. ST Phys. Educ. Res. 9, 020119 (2013). 
[10] L. Bollen, P. van Kampen, and M. De Cock, Students' difficulties with vector calculus in electrodynamics, Phys. Rev. ST Phys. Educ. Res. 11, 020129 (2015).

[11] C. Singh, Student understanding of quantum mechanics, Am. J. Phys. 69, 885 (2001).

[12] E. Marshman and C. Singh, Framework for understanding student difficulties in quantum mechanics, Phys. Rev. ST Phys. Educ. Res. 11, 020119 (2015).

[13] C. Singh and E. Marshman, Review of student difficulties in quantum mechanics, Phys. Rev. ST Phys. Educ. Res. 11, 020117 (2015).

[14] M. C. Wittmann, R. N. Steinberg, and E. F. Redish, Investigating student understanding of quantum physics: Spontaneous models of conductivity, Am. J. Phys. 70, 218 (2002).

[15] S. B. McKagan, K. K. Perkins, and C. E. Wieman, Deeper look at student learning of quantum mechanics: The case of tunneling, Phys. Rev. ST Phys. Educ. Res. 4, 020103 (2008).

[16] E. Cataloglu and R. W. Robinett, Testing the development of student conceptual and visualization understanding in quantum mechanics through the undergraduate career, Am. J. Phys. 70, 238 (2002).

[17] T. Wan, P. J. Emigh, and P. S. Shaffer, Investigating how students relate inner products and quantum probabilities, Phys. Rev. Phys. Educ. Res. 15, 010117 (2019).

[18] B. W. Dreyfus, A. Elby, A. Gupta, and E. R. Sohr, Mathematical sense-making in quantum mechanics: An initial peek, Phys. Rev. Phys. Educ. Res. 13, 020141 (2017).

[19] E. R. Sohr, A. Gupta, A. Elby, A. Gupta, and B. W. Dreyfus, Sense-making with inscriptions in quantum mechanics, in Proceedings of the 2016 Physics Education Research Conference, Sacramento, CA (AIP, New York, 2016).

[20] G. Zhu and C. Singh, Improving students' understanding of quantum mechanics via the Stern-Gerlach experiment, Am. J. Phys. 79, 499 (2011).

[21] G. Passante, P. J. Emigh, and P. S. Shaffer, Student ability to distinguish between superposition states and mixed states in quantum mechanics, Phys. Rev. ST Phys. Educ. Res. 11, 020135 (2015).

[22] R. Sayer, A. Maries, and C. Singh, Quantum interactive learning tutorial on the double-slit experiment to improve student understanding of quantum mechanics, Phys. Rev. Phys. Educ. Res. 13, 010123 (2017).

[23] M. Michelini and G. Zuccarini, University Students' Reasoning on Physical Information Encoded in Quantum State at a Point in Time, in Proceedings of the 2014 Physics Education Research Conference, Minneapolis, MN (AIP, New York, 2014), pp. 187-190.

[24] P. R. L. Heron, Identifying and Addressing Difficulties: Reflections on the Empirical and Theoretical Basis of an Influential Approach to Improving Physics Education, in Getting Started in PER, Reviews in PER, edited by C. Henderson and K. A. Harper (American Association of Physics Teachers, College Park, MD, 2018), Vol. 2.

[25] P. R. L. Heron, Empirical investigations of student understanding, in Proceedings of the 2003 Physics Education Research Conference, Madison, WI (AIP, New York, 2003).
[26] V. Braun and V. Clarke, Using thematic analysis in psychology, Qual. Res. Psychol. 3, 77 (2006).

[27] http://www.biostathandbook.com/fishers.html.

[28] Q. McNemar, Note on the sampling error of the difference between correlated proportions or percentages, Psychometrika 12, 153 (1947).

[29] https://studentdata.washington.edu/quick-stats/.

[30] D. H. McIntyre, Quantum Mechanics: A Paradigm Approach, 1st ed. (Addison-Wesley, Boston, MA, 2012).

[31] L. C. McDermott, P. S. Shaffer, P. R. L. Heron (the Physics Education Group), Tutorials in Physics: Quantum Mechanics (to be published).

[32] D. J. Griffiths, Introduction to Quantum Mechanics (Pearson Prentice Hall, Upper Saddle River, NJ, 2005).

[33] A. Gupta and A. Elby, Beyond epistemological deficits: Dynamic explanations of engineering students' difficulties with mathematical sense-making, Int. J. Sci. Educ. 33, 2463 (2011).

[34] The ranges are, from top quartile to bottom quartile, 30\%$35 \%, 20 \%-35 \%, 20 \%-30 \%$, and $10 \%-20 \%$. All numbers are rounded to the nearest $5 \%$.

[35] E. M. Smith, J. P. Zwolak, and C. A. Manogue, Student difficulties with complex numbers, Proceedings of the 2015 Physics Education Research Conference, College Park, MD (AIP, New York, 2015).

[36] T. Wan, P. J. Emigh, and P. S. Shaffer, Student understanding of the measurable effects of relative phases in superposition states, Proceedings of the 2017 Physics Education Research Conference, Cincinnati, OH (AIP, New York, 2017), pp. 420-423.

[37] P. J. Emigh, G. Passante, and P. S. Shaffer, Student understanding of superposition: Vectors and wave functions, in Proceedings of the 2016 Physics Education Research Conference, Sacramento, CA (AIP, New York, 2016).

[38] M. E. Loverude, Mathematization and the 'Boas course', Proceedings of the 2017 Physics Education Research Conference, Cincinnati, OH (AIP, New York, 2017).

[39] G. Passante, Energy measurement resources in spins-first and position-first quantum mechanics, in Proceedings of the 2016 Physics Education Research Conference, Sacramento, CA (AIP, New York, 2016).

[40] D. Hammer, Student resources for learning introductory physics, Am. J. Phys. 68, S52 (2000).

[41] D. Kahneman, Thinking, Fast and Slow (Farrar, Straus, and Girous, New York, 2011).

[42] C. R. Gette and M. Kryjevskaia, Establishing a relationship between student cognitive reflection skills and performance on physics questions that elicit strong intuitive responses, Phys. Rev. Phys. Educ. Res. 15, 010118 (2019).

[43] M. Kryjevskaia, M. R. Stetzer, and N. Grosz, Answer first: Applying the heuristic-analytic theory of reasoning to examine student intuitive thinking in the context of physics, Phys. Rev. ST Phys. Educ. Res. 10, 020109 (2014).

Correction: The word "superposition" was unintentionally transformed to another word in the sentence in Sec. I that begins "Passante et al." during the production process and has been set right. 